Review Article

\title{
Comparative Effectiveness of Anti-Inflammatory Drug Treatments in Coronary Heart Disease Patients: A Systematic Review and Network Meta-Analysis
}

\author{
Ivan Wudexi, Elica Shokri, Mohamed Abo-Aly, Kazuhiro Shindo, and Ahmed Abdel-Latif \\ Gill Heart Institute and Division of Cardiovascular Medicine, University of Kentucky and the Lexington VA Medical Center, \\ Lexington, KY, USA \\ Correspondence should be addressed to Ahmed Abdel-Latif; abdel-latif@uky.edu
}

Received 5 August 2020; Accepted 30 December 2020; Published 15 January 2021

Academic Editor: Helen C. Steel

Copyright ( 2021 Ivan Wudexi et al. This is an open access article distributed under the Creative Commons Attribution License, which permits unrestricted use, distribution, and reproduction in any medium, provided the original work is properly cited.

\begin{abstract}
Introduction and Hypothesis. The role of inflammation is widely recognized in the pathogenesis of coronary artery disease. Research on animal models had shown the potential benefits of targeting specific inflammatory pathways. However, studies on human subjects are limited with small number of patients and no head-to-head comparisons. Methods. We conducted a network metaanalysis of randomized controlled trials that studied the effects of anti-inflammatory medications on cardiovascular outcomes of coronary artery disease patients. We searched the electronic database until March 2020 for relevant studies. Results. Nineteen trials examining the efficacy of eight anti-inflammatory medications (pexelizumab, anakinra, colchicine, darapladib, varespladib, canakinumab, inclacumab, and losmapimod) were selected for analysis. Overall, there is no statistically significant difference in all-cause mortality, cardiovascular mortality, revascularization, and major cardio and cerebrovascular events (MACCE) with the use of anti-inflammatory drugs. However, we found the use of colchicine significantly reduces the odds of developing stroke by approximately $75 \%$ (OR 0.26, CI 0.10-0.63). Colchicine use was also associated with a lower risk of revascularization and MACCE compared to the other agents. Our subgroup analyses comparing the timing of medication initiation (within 7 days vs. $>7$ days) and clinical presentation (ACS vs. non-ACS) revealed a significant reduction in the risk of recurrent MI in the group that received medication after seven days (OR 0.92, CI 0.86-0.99) and the non-ACS group (OR 0.88, CI 0.80-0.98). Conclusion. Although many anti-inflammatory medications have failed to reduce adverse cardiovascular outcomes in the CAD population, selected medications show promise among subgroups of patients without ACS or after the first week following an acute ischemic event. Future studies examining the proper timing and targetable anti-inflammatory pathways are warranted.
\end{abstract}

\section{Introduction}

Each year, roughly 1.1 million patients are hospitalized with an acute coronary syndrome (ACS) such as an acute myocardial infarction (AMI) event in the United States [1]. Despite the use of optimal guideline, directed medical therapies and secondary prevention, recurrent ischemic coronary events, and mortality remain high among patients with coronary artery disease, with an annual rate of 4 to $5 \%$ after their initial ischemic event. Accordingly, ischemic heart disease remains the leading cause of heart failure and mortality in the western world. Over the past 2 decades, there has been an increasing interest in discovering therapeutic agents for reducing resid- ual risk among patients with acute coronary syndromes (ACS), including ST segment elevation myocardial infarction (STEMI), non-ST segment myocardial infarction (NSTEMI), and unstable angina $[2,3]$.

Decades of animal and human research have confirmed the critical role that inflammation plays in the development and progress of atherosclerosis. Innate immune cells such as neutrophils and proinflammatory monocytes play a critical role in the body's response to sterile tissue injury such as after myocardial infarction [4]. While the immune response is necessary for clearing dead cells and preparing the myocardium for healing, exacerbated inflammation, as often seen in mammals, can lead to detrimental effects. 
Studies have shown strong correlation between elevated neutrophil and monocyte count and infarction expansion as well as poor clinical outcomes after AMI [5-8]. Furthermore, attempts at modulating the immune response after AMI have resulted in attenuated myocardial damage, reduced atherosclerosis burden, and enhanced survival [9-11]. This increased interest in targeting inflammation using clinically relevant therapies.

Modulating the inflammatory response post-AMI is an elusive target as studies have shown that complete systemic suppression of inflammation is rather harmful [12]. Delayed healing and ventricular aneurysms were reported with glucocorticosteroids [13]. Similarly, nonsteroidal antiinflammatory drug use in coronary artery disease (CAD) patients is associated with higher mortality and recurrent AMI [14]. Additionally, studies aimed at depleting inflammatory cells failed to demonstrate benefit [15]. On the other hand, selective targeting of inflammatory mediators such as IL- $1 \beta$ using selective monoclonal antibodies demonstrated success in clinical studies, albeit with high cost and modest benefit [16-18]. Therefore, strategies aimed at modulating the inflammatory response rather than its suppression provide therapeutic promise. However, studies using selective targets of the inflammatory pathways in CAD patients remain small and underpowered to reach conclusions. Furthermore, with multiple new targeted anti-inflammatory agents (referred to as anti-inflammatory drugs throughout the manuscript), agents being studies in the field, there are no head-to-head comparisons between and conducting such a comparison using the randomized design will be prohibitively expensive. Studies on human subjects were limited to a small study sample and no head-to-head comparisons [19]. In this meta-analysis, we seek to perform a cumulative analysis of the efficacy of new anti-inflammatory drugs to reduce clinical events among CAD patients. We also performed a network meta-analysis to investigate the efficacy and safety between these agents.

\section{Materials and Methods}

We conducted this protocol-driven systematic review and meta-analysis according to the Preferred Reported Items for Systematic Reviews and Meta-Analyses (PRISMA) [20]. We systematically searched PubMed, Cochrane, and Scopus for relevant studies through March 2020. The search formula for each database is provided in detail in the online supplementary appendix (available here). We also screened the references of relevant meta-analysis and systematic reviews for eligible studies. The abstracts of the American Heart Association, American College of Cardiology, and European Society of Cardiology were screened over the last 2 years for eligible studies.

We included randomized controlled trials (RCTs) that studied the effects of the following anti-inflammatory medications on cardiovascular outcomes of patients with coronary artery disease: pexelizumab, colchicine, darapladib, varespladib, anakinra, canakinumab, inclacumab, and losmapimod. Our primary cardiovascular outcomes of interest were allcause death, cardiovascular death, recurrent myocardial infarction, stroke, revascularization, and major adverse cardiac and cerebrovascular events (MACCE), defined as a composite of death/cardiovascular death, myocardial infarction, and stroke. In studies that did not report this definition of MACCE, we accepted the closest composite to our MACCE definition. In studies that use multiple dosages of a medication, we use the highest dose except when data is only available for a lower dose across multiple studies of the same medication. In studies that reported clinical outcomes at multiple follow-up lengths, we included the data from the longest available follow-up duration. We excluded studies that did not have the cardiovascular outcomes of interest or enrolled patients with significant medical comorbidities that may affect the outcomes (e.g., cancer patients who receive chemotherapy).

Two reviewers (E.S and I.W) independently screened and evaluated the eligibility of the studies using the aforementioned criteria. Studies were initially screened by title and abstract. After initial screening, the full text of the identified studies was reviewed thoroughly to determine their eligibility. Baseline characteristics and clinical outcomes of interest were extracted using a standardized data extraction form. Disagreements were resolved through discussions, and the opinion of a third investigator (M.A.) was requested if necessary. Quality assessment of the studies was performed using the Cochrane Collaboration's risk of bias tool [21].

2.1. Statistical Analyses. The prespecified outcomes of our analyses were all-cause mortality, cardiac mortality, recurrent myocardial infarction, stroke, revascularization, and major adverse cardiac and cerebrovascular events (MACCE). Summary estimates were calculated as odds ratios (OR) with 95\% confidence intervals (CI) using the random-effects model based on DerSimonian and Laird's meta-analytic statistical method [22]. Considering the heterogeneity of the included trials and its potential influence on treatment effects, we prespecified the use of random-effects model to assess effect sizes. The $I^{2}$ index was used to summarize the proportion of total variability in the estimate. The $I^{2}$ statistic is derived from the $Q$ statistic and describes the percentage of total variation across studies attributed to heterogeneity; values of $25 \%, 50 \%$, and $75 \%$ correspond to low, moderate, and high heterogeneity, respectively $[23,24]$. We performed subgroup analyses according to (a) type of enrolled population in studies (stable coronary heart disease and CABG vs. ACS) and (b) the duration between symptom onset/index event and study medication administration. We used visual inspection of funnel plots to assess for publication bias. The statistical level of significance was 2 -tailed $P<0.05$. Analyses were performed using Review Manager version 5.3 (Revman; The Cochrane Collaboration, Oxford, UK).

In the network meta-analysis, we used the Bayesian Markov chain Monte Carlo modelling using the informative prior setting. The Bayesian model was selected because of its greater flexibility and ability to rank treatments according to their comparative effectiveness. Informative prior was chosen to assume consistency between heterogeneity variances and ensure a realistic heterogeneity estimation [25]. All network analyses were performed with NetMetaXL 1.6.1 


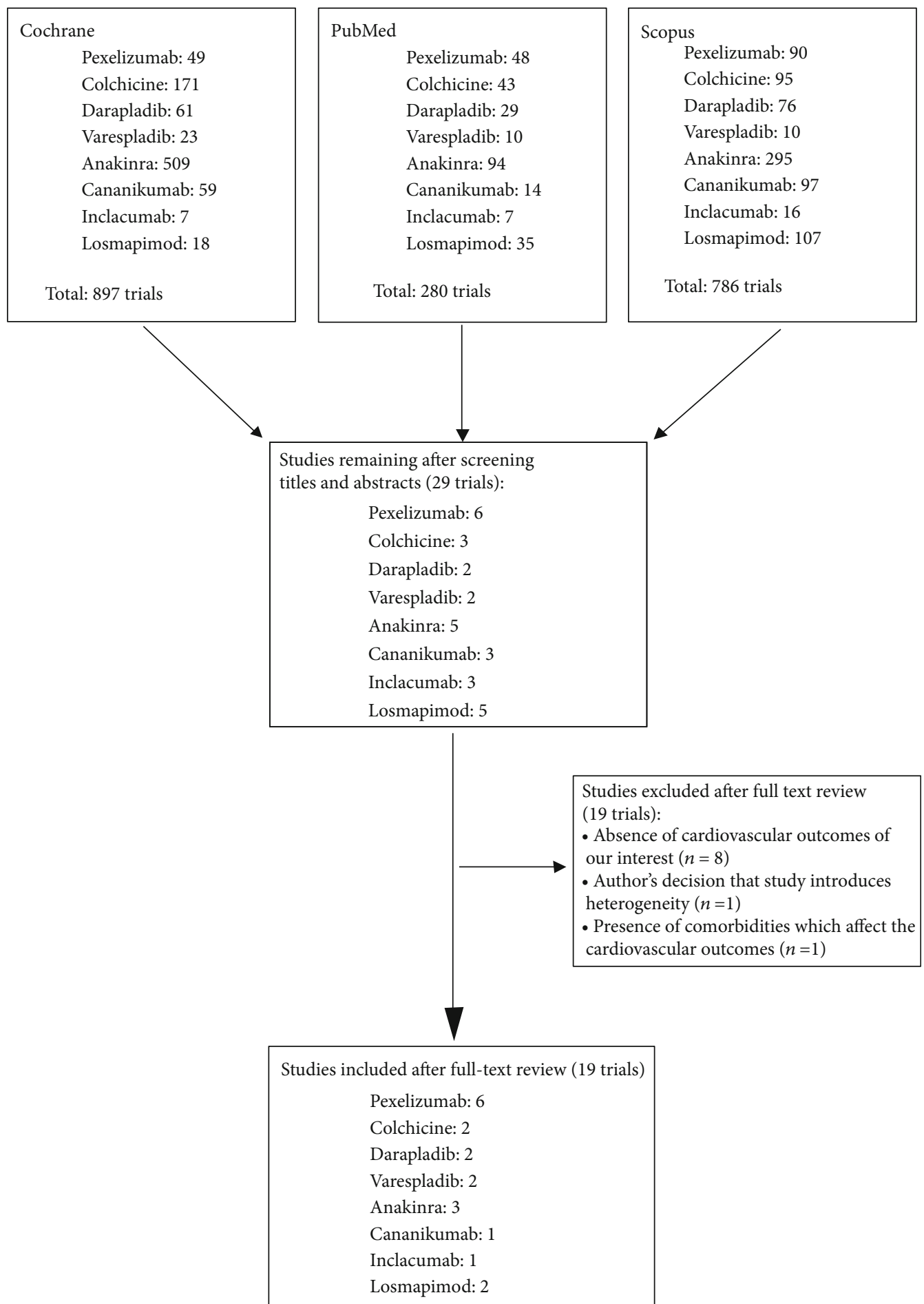

Figure 1: Flow chart showing the search algorithm and final study selection in the meta-analysis.

(Canadian Agency for Drugs and Technologies in Health, Ottawa, Canada) and WinBUGS 1.4.3 (MRC Biostatistics Unit, Cambridge, United Kingdom) [26]. A burn-in phase of 20,000 iterations was used to achieve convergence. The convergence was assessed using the Brooks-Gelman-Rubin plots. NetMetaXL fits three chains for Bayesian network meta-analysis. We evaluated heterogeneity using the between-study heterogeneity variances per outcome, known as $\tau^{2}$ (tau-squared) and its $95 \% \mathrm{CI}$. Inconsistency was evaluated by plotting the posterior mean deviation of individual data points in the inconsistency model against its posterior mean deviation in the consistency model to identify any loop in the treatment network where inconsistency exists [27]. 


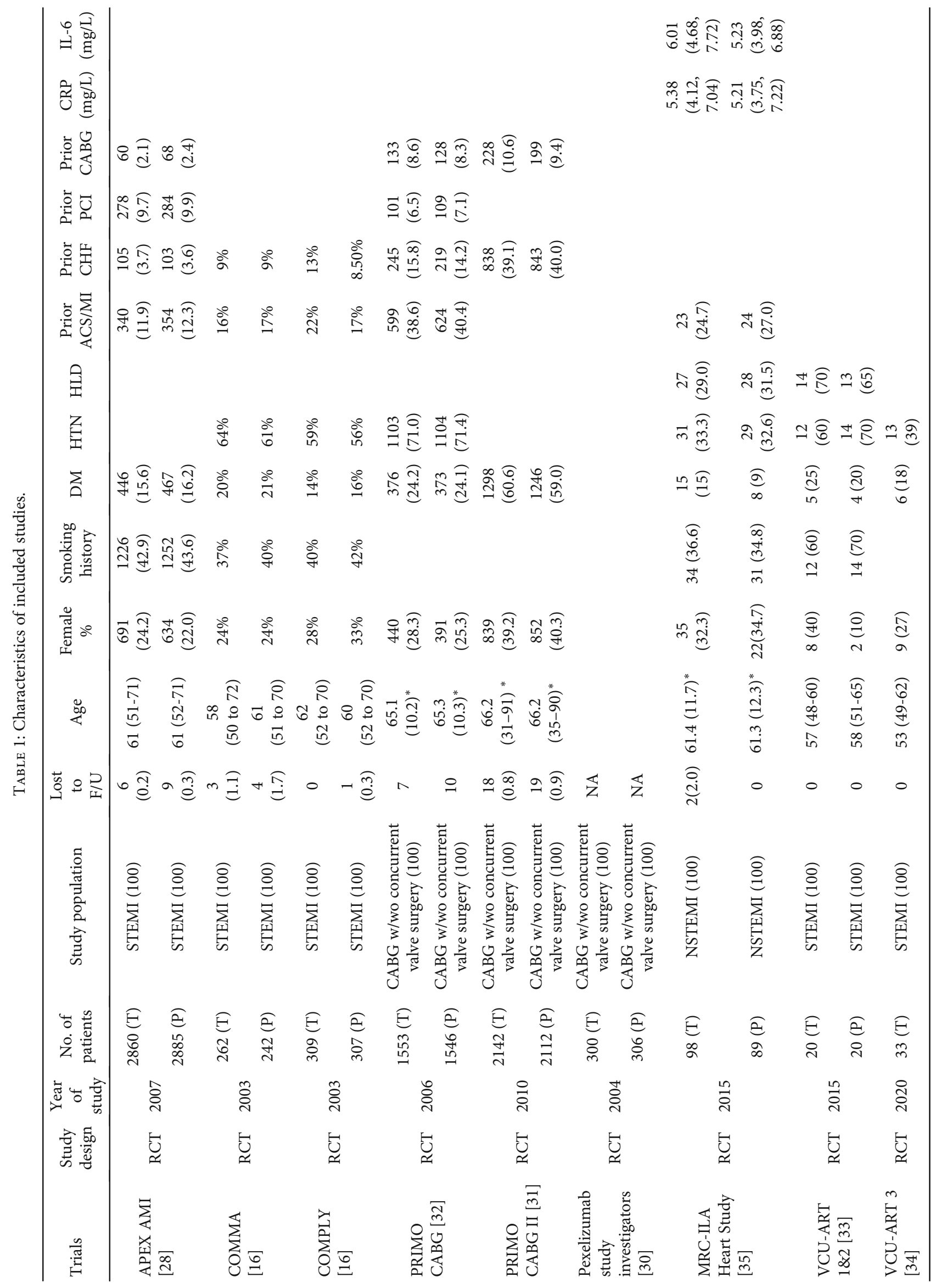




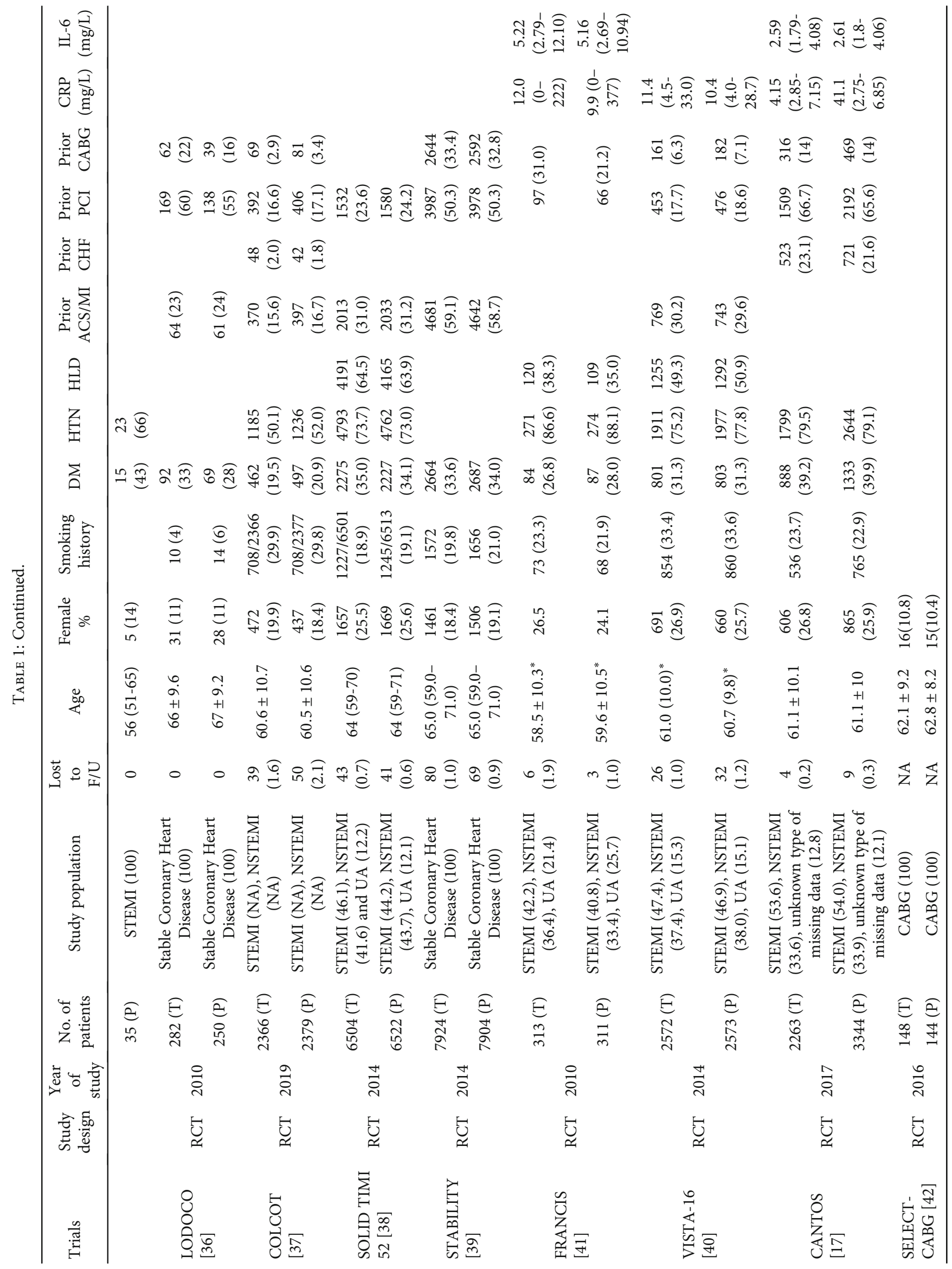




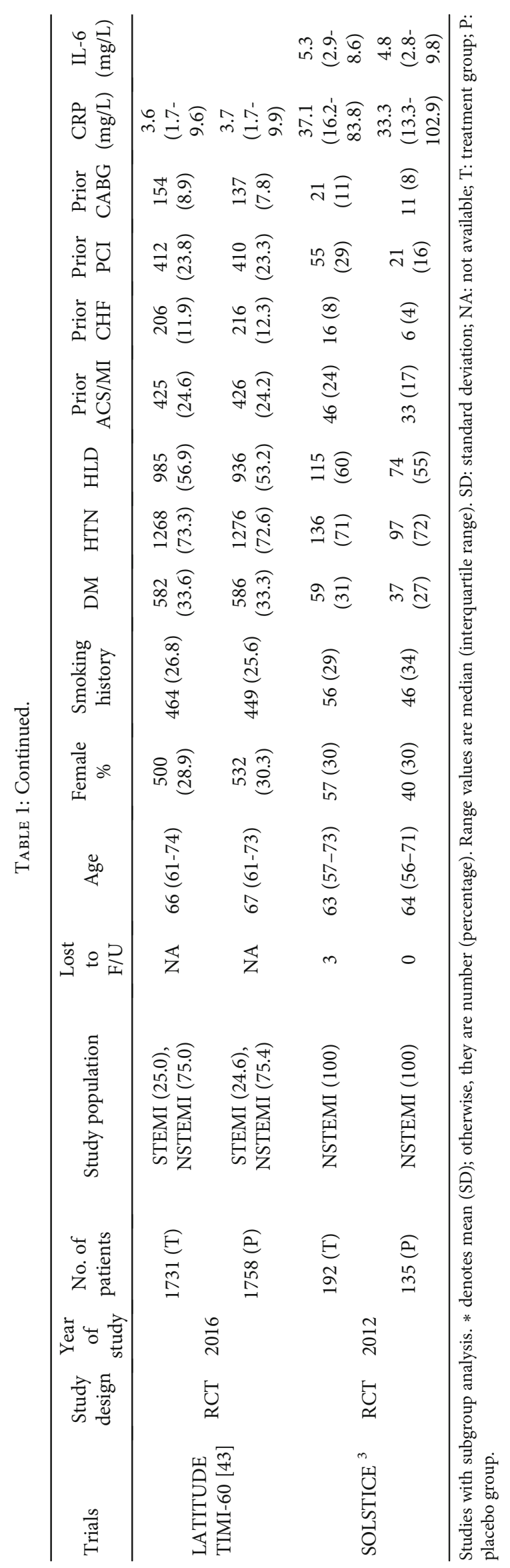


TABLE 2: Patient population and clinical scenario among included studies.

\begin{tabular}{lcc}
\hline Patient population & Study & Investigational drug \\
\hline & APEX-MI [28] & Pexelizumab \\
STEMI & COMMA [16] & COMPLY [16] \\
& VCU-ART3 [34] & Anakinra \\
NSTEMI & MRC-ILA Heart Study [35] & Canakinumab \\
& CANTOS [17] & Cosmapimod \\
MI (STEMI and NSTEMI) & LATITUDE TIMI 60 [43] & Darapladib \\
& COLCOT [37] & Varespladib \\
ACS (STEMI, NSTEMI, or UA) & SOLID TIMI 52 [38] & Varespladib \\
& FRANCIS [41] & PISTA-16 [40] \\
CABG & PRIMO CABG 1 [32] & PRIMO CABG 2 [31] \\
Stable CAD & Pexelizumab Study Investigators [30] & Inclacumab \\
\end{tabular}

*Study with subgroup analysis of its primary end points.

\section{Results}

Our literature search yielded a total of 1963 trials, as shown in Figure 1. After title and abstract screening and full-text review, nineteen trials including 70,620 patients met our inclusion criteria utilizing the following medications: pexelizumab (6 studies) [16, 28-32], anakinra (3 studies) [33-35], colchicine (2 studies) [36, 37], darapladib (2 studies) [38, 39], varespladib (2 studies) [40, 41], canakinumab (1 study) [17], inclacumab (1 study) [42], and losmapimod (2 studies) $[3,43]$. The baseline characteristics of the study population are shown in Table 1 . The median patient age ranges from 53-67 years old, $77.3 \%$ of the patient population were males, and the follow-up duration ranged from 30 days to 3.7 years. Overall, patients included in this meta-analysis had the typical comorbidities of this cohort including diabetes, hypertension, hyperlipidemia, and high prevalence of smoking. Population of patients included in our meta-analysis ranged from chronic CAD (34.8\%), patients undergoing CABG (11.7\%), and ACS patients (57\%). Of the total number of 19 studies, 6 trials enrolled stable coronary artery disease [30-32, 36, 39, 42] patients, 3 trials investigated ACS patients with spectrum of unstable angina to STEMI [38, 40, 41], 3 trials enrolled patients with either STEMI or NSTEMI [17, 37, 43], 5 studies only included patients who presented with STEMI [16, 28, 29, 33, 34], and 2 trials enrolled only NSTEMI patients $[3,35]$.

The breakdown of the trials with the corresponding patient populations is shown in Table 2. The quality assessment of the included studies is displayed in Supplemental Table 1 of the Appendix.

3.1. Meta-Analysis Results. There was a reduction in the risk of revascularization (OR 0.85, CI 0.73-1.00; $P=0.04$ ) with the use of anti-inflammatory drugs compared to standard of care alone (Figure 2). However, there was no statistically significant difference in cardiovascular mortality (OR 0.93, CI $0.84-1.02 ; P=0.13$ ), all-cause mortality (OR 0.96 , CI $0.87-1.05 ; P=0.38$ ), stroke (OR 0.96, CI 0.82-1.13; $P=0.65$ ), recurrent myocardial infarction (OR 0.99, CI 0.89-1.10; $P=0.82$ ), and major adverse cardio and cerebrovascular events (MACCE) (OR 0.95, CI 0.87-1.04; $P=0.24$ ) with the use of anti-inflammatory medications (Figures 3-7).

While we did not observe a significant reduction in the incidence of stroke with the use of anti-inflammatory drugs, we found the use of colchicine significantly reduces the stroke odds by approximately $75 \%$ in patients with coronary artery disease (OR 0.26, CI 0.10-0.63; $P=0.003$ ) (Figure 5). On the other hand, we found that the use of anakinra was associated with an almost fourfold increase in the risk of developing recurrent MI (OR 3.85, CI 1.04-14.28; $P=0.04$ ) (Figure 6). Lastly, although the PRIMO CABG trial had previously shown that pexelizumab treatment improves MACCE in patients undergoing CABG, our pooled analysis showed only a trend in the reduction of MACCE with pexelizumab compared to placebo (OR 0.93, CI 0.84-1.02; $P=0.12$ ) (Figure 7).

\subsection{Subgroup Analysis}

3.2.1. Acute vs. Subacute vs. Chronic Presentation. The results of the subgroup analyses according to time of study medication initiation after symptom onset/index event ( 7 days vs. $>7$ days) are summarized in Table 3. Patients who were given study medications after 7 days of index ischemic event demonstrated reduced risk for developing recurrent $\mathrm{MI}$ and revascularization $(P$ value for interaction $=0.03)$. There was also a trend in favor of drug initiation after 7 days from symptom onset or index clinical event in all-cause mortality, cardiac mortality, and MACCE. 


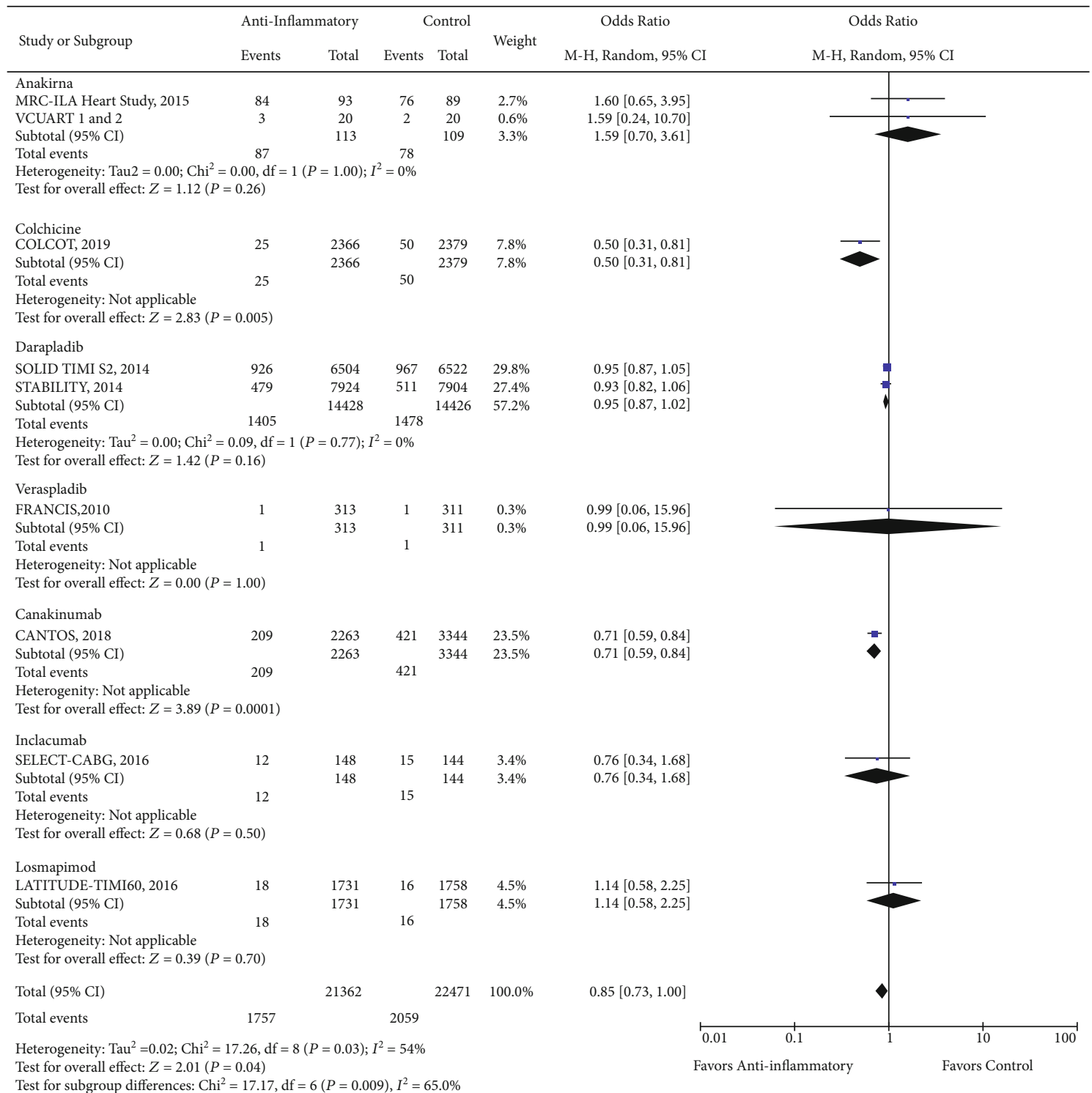

FIGURE 2: Forest plot for the comparative risk of revascularization with anti-inflammatory therapy versus standard of care alone. Antiinflammatory therapy significantly reduced the risk of revascularization (OR 0.85, CI 0.73-1.00; $P<0.05)$.

3.3. ACS vs. Non-ACS Presentation. The sensitivity analysis based on clinical presentation (ACS vs. non-ACS) is summarized in Table 3 . In our study, the use of anti-inflammatory medications was found to reduce the risk of recurrent MI in the non-ACS population compared to the ACS population (OR 0.88, CI 0.80-0.98; $P=0.04$ ). There was also a trend in favor of starting anti-inflammatory drugs in non-ACS patients when MACCE was assessed. Meanwhile, no significant differences in other cardiovascular outcomes (e.g., allcause mortality, CV death, stroke, and recurrent $\mathrm{MI}$ ) were noted in our analyses.

3.4. Network Meta-Analysis. Supplemental Figures 1-6 show the forest plot comparing the relative efficacies of each anti-inflammatory medication on all-cause mortality, cardiovascular death, recurrent myocardial infarction, revascularization, stroke, and MACCE. We found the use of colchicine was significantly associated with reduced risk of revascularization, stroke, and MACCE in comparison with several anti-inflammatory medications in our study. Specifically, colchicine was associated with lower risk of revascularization events than both anakinra and darapladib (OR 0.31, CI 0.11-0.84 and OR 0.52, CI 0.29-0.93). It was also associated with lower risk of stroke after MI when compared with the use of darapladib, pexelizumab, losmapimod, canakinumab, and varespladib (OR 0.23, CI 0.07-0.57; OR 0.23, CI 0.07-0.64; OR 0.25, CI 0.07-0.85; OR 0.30, CI 0.09-0.81; and OR 0.26, CI 0.07-0.97, respectively). Furthermore, colchicine use was also associated with lower risk of MACCE compared to darapladib, losmapimod, anakinra, or varespladib (OR 0.69, CI 0.44-0.98; OR 0.60, CI 0.37-0.93; OR 0.28, CI 0.10-0.70; and OR 0.53, CI 0.320.83 , respectively). Visualization of the network metaanalysis is depicted in Supplemental Figure 7. 


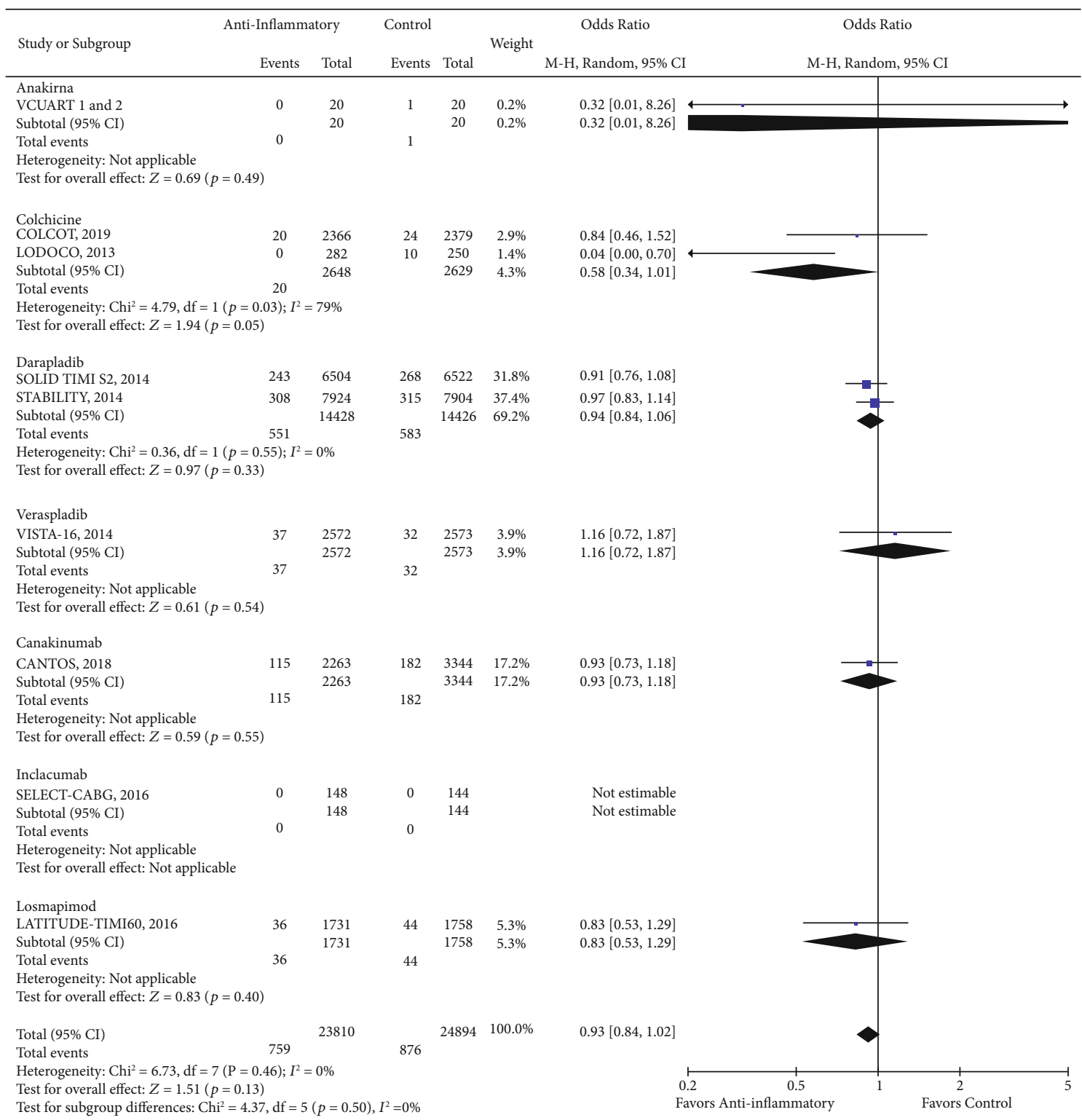

FIGURE 3: Forest plot of cardiac mortality. There was no significant difference between anti-inflammatory therapy and standard therapy alone regarding cardiovascular mortality (OR 0.93, CI 0.84-1.02; $P=0.13$ ).

3.5. Assessment of Heterogeneity. We drew funnel plots to seek evidence of publication bias: where inconsistency was high, the funnel plots were not interpretable; where inconsistency was low, the funnel plots were inconclusive (Supplemental Figure 8). In the network meta-analysis, we did not observe significant inconsistency in our analysis of the outcomes (Supplemental Figure 9).

\section{Discussion}

The use of selective anti-inflammatory drugs to reduce the incidence of cardiovascular events in high-risk patients with CAD is debatable in the clinical community. We conducted a systematic review and network meta-analysis to study the effect of several anti-inflammatory medications on cardiovascular outcomes in coronary artery disease patients. In our systematic review of eight anti-inflammatory medica- tions, we observed a modest reduction in cardiovascular outcomes when compared with placebo. However, as previously published, the use of colchicine and canakinumab did show a beneficial effect on reducing the risk of revascularization post-AMI [17, 37]. Furthermore, there was a significant reduction in the incidence of stroke in the CAD population who received colchicine as compared with the placebotreated group. Colchicine use was also associated with lower odds of MACCE compared to other selective antiinflammatory agents.

Our subgroup analyses based on the timing of medication initiation ( $<7$ days vs. $>7$ days) and patient's clinical presentation (ACS vs. non-ACS) demonstrated interesting and unexpected results. Theoretically, the administration of anti-inflammatory agents is expected to demonstrate benefit during heightened inflammation after ACS. However, our subgroup analyses suggest that anti-inflammatory drugs 


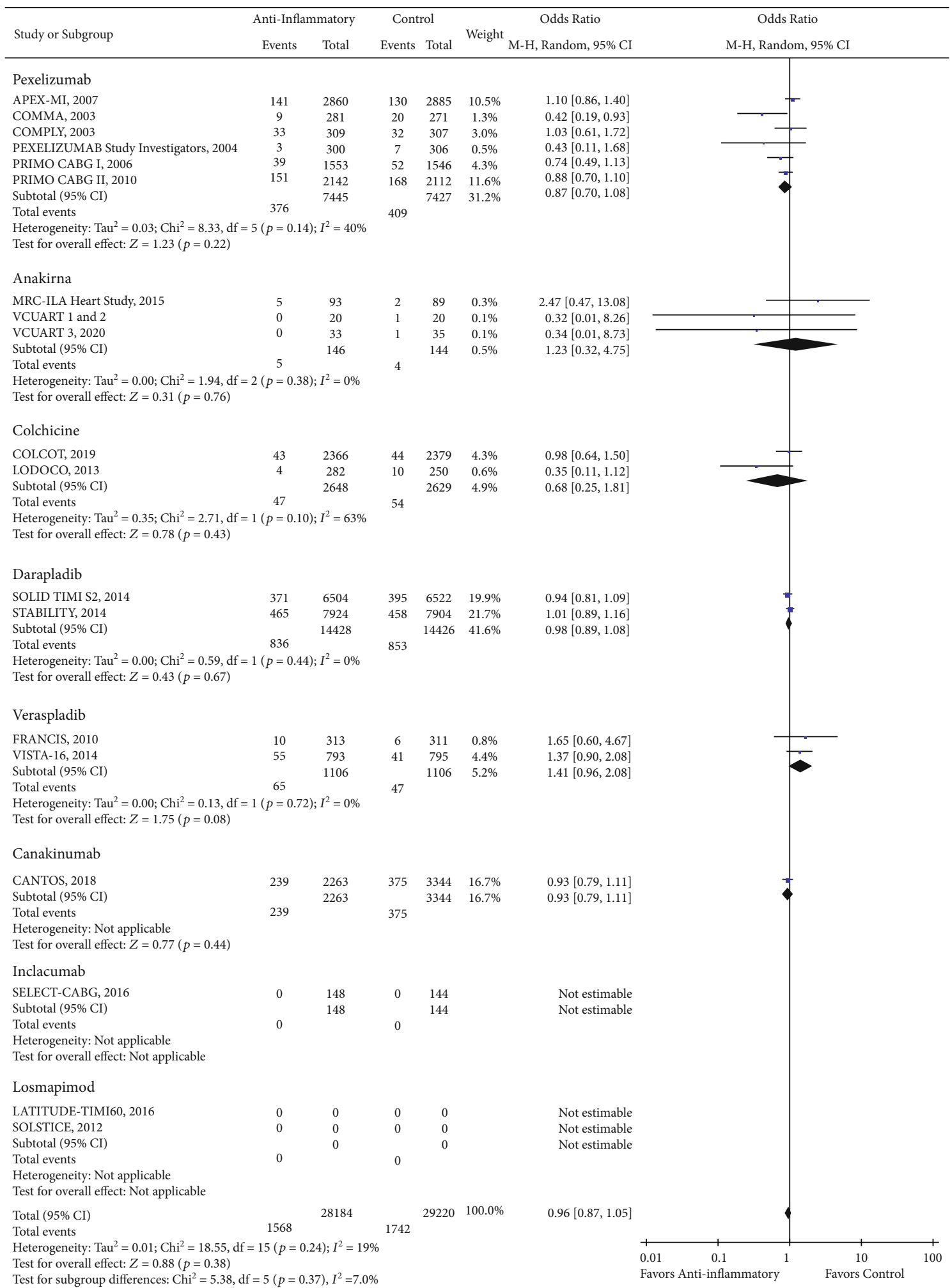

Figure 4: Forest plot of all-cause mortality. No significant difference was observed in the risk of all-cause mortality between antiinflammatory therapy and standard therapy alone (OR 0.96, CI 0.87-1.05; $P=0.38$ ).

could potentially be more beneficial among patients with non-ACS presentation and those initiated on therapy after 7 days from symptom onset. This could be explained by the physiological importance of the nonsterile inflammatory response early after coronary ischemia [12]. It has been dem- onstrated in animal studies that depleting inflammatory cells such as neutrophils and macrophages after AMI is rather detrimental and could result in increased mortality and exacerbation of heart failure. To our knowledge, there has not been a human study that directly compares the efficacy of 


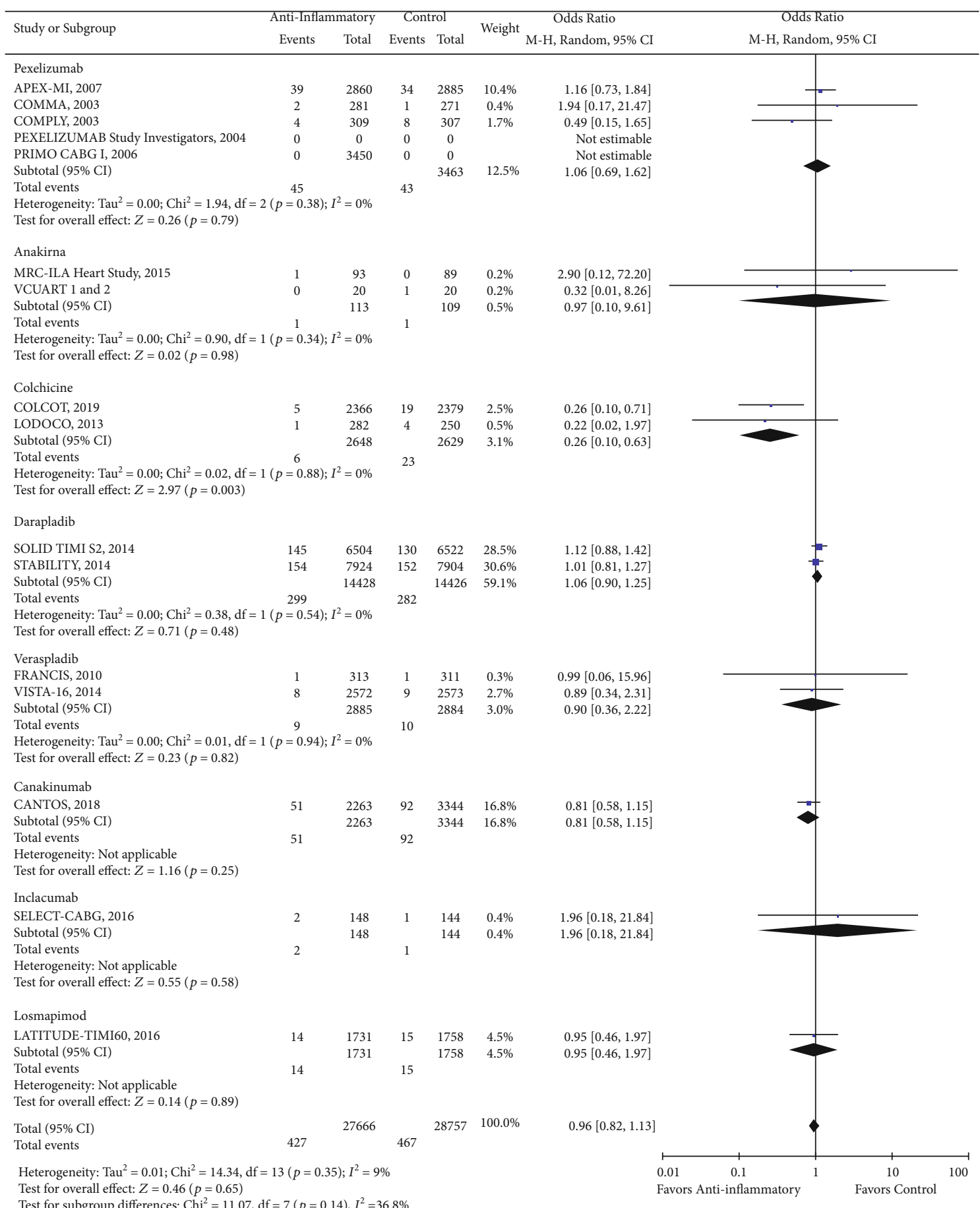

FIGURE 5: Forest plot of stroke. There was no significant difference between anti-inflammatory therapy and standard therapy alone in regard to stroke (OR 0.96, CI 0.82-1.13; $P=0.65$ ).

anti-inflammatory medications given at different time points after index events. We believe future studies that compare the timing of medication administration and its association with cardiovascular outcomes are necessary to further explore the underpinnings of this phenomenon.

4.1. Colchicine and Stroke Incidence. Our study finding was consistent with a recently published meta-analysis study that looked into the efficacy of colchicine in preventing stroke in patients with coronary artery disease. Even though our analysis excluded two studies included in this meta-analysis, due to the absence of cardiovascular outcomes as either primary or secondary endpoints, a similar degree of stroke risk reduction was demonstrated (OR 0.31, 95\% confidence interval: $0.13-0.71 ; P=0.006)$ [44]. The proposed mechanisms of action of colchicine are through its effect on neutrophil and monocytes through inhibition of NLRP3 inflammasome complex formation and microtubule function, which inhibits 


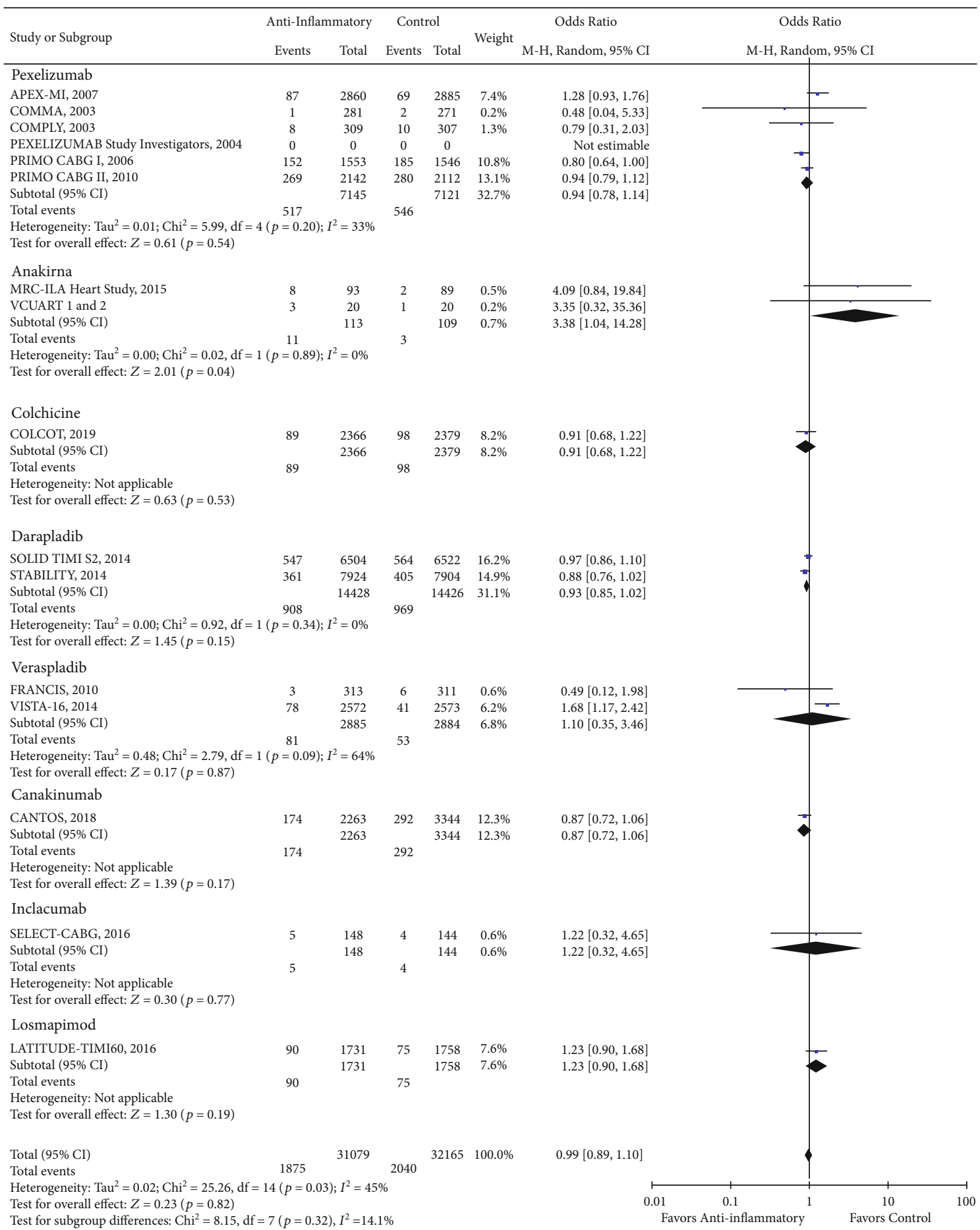

Figure 6: Forest plot of recurrent myocardial infarction. No significant difference was observed between anti-inflammatory therapy and standard therapy in terms of recurrent myocardial infarction (OR 0.99, CI 0.89-1.10; $P=0.82$ ).

the production of IL- 1 beta and IL-18 and prevents the migration of inflammatory cells, respectively. Inhibition of these pathways had been shown to decrease both hsCRP levels and atherosclerotic plaque progression as well as instability on CT scan [45]. It is worth noting that the pooled clinical benefit seen with the use of colchicine in our study largely derived from the study population with acute myocardial infarction as opposed to stable coronary disease [36, 37]. It is possible that this finding is related to higher transcoronary gradients of IL-1 and IL-18 seen in the ACS than the stable CAD population [46]. Thus, inhibition of these interleukins by colchicine might explain the reduced risk of stroke in patients with recent $\mathrm{MI}$, as had been demonstrated in previous RCT [37]. 


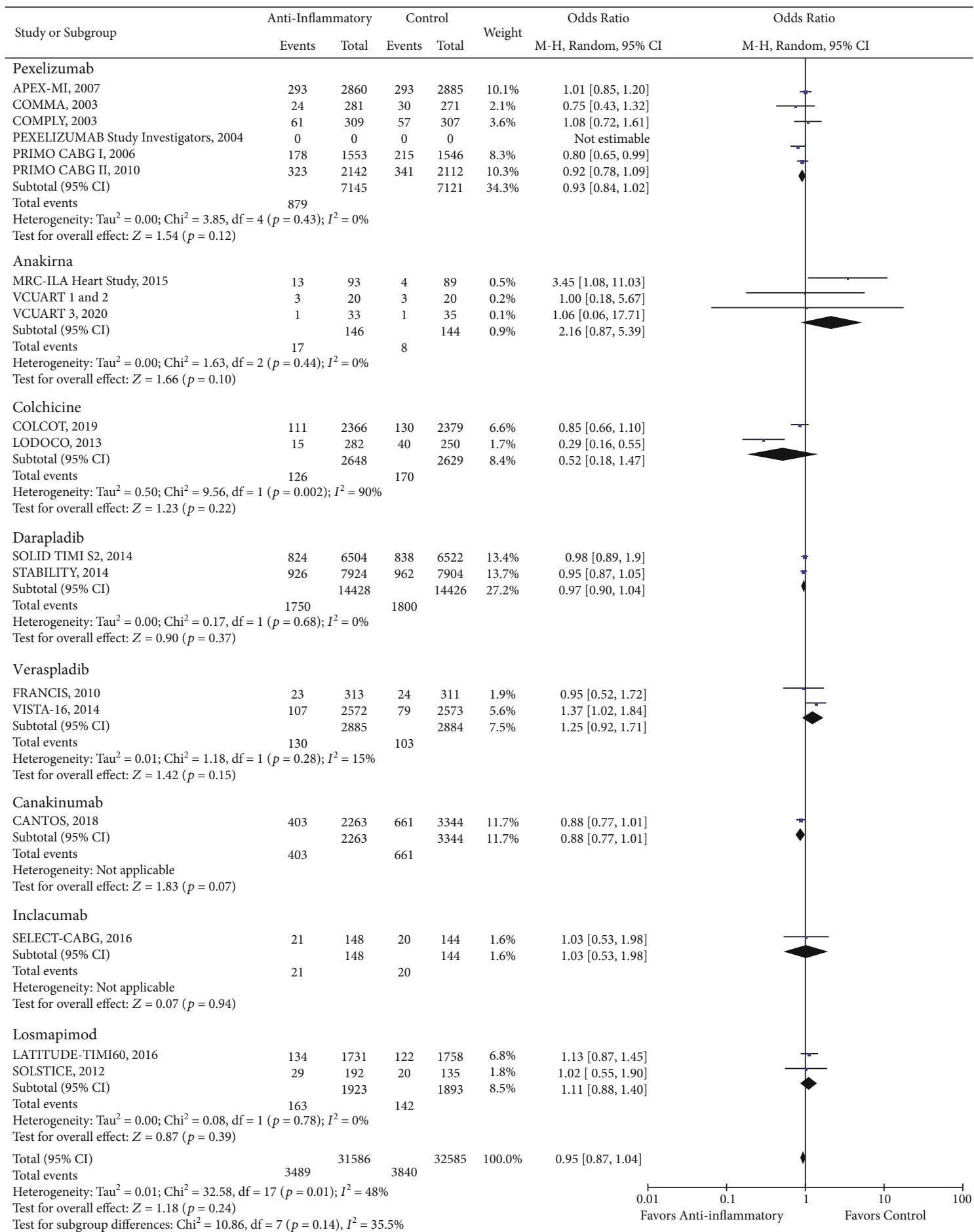

Figure 7: Forest plot of MACCE. There was no significant difference between anti-inflammatory therapy and standard therapy alone regarding MACCE (OR 0.95, CI 0.87-1.04; $P=0.24$ ).

4.2. Anakinra and Recurrent MI. Our findings are consistent with a recent meta-analysis that studied the effect of IL-1 blockage on cardiovascular risk. The authors found an overall increased risk of recurrent MI with anakinra after pooling data from a total of five anakinra trials [47]. Although we only included three of the five trials based on our prespecified inclusion criteria (we excluded one study that included only the heart failure population and another study that did not have cardiovascular outcome as either primary or secondary end points) in our analysis, we found similar risk estimates with a wide confidence interval. This finding is in contrast with the CANTOS trial where the use of another IL-1 blockade medication, canakinumab, had resulted in decreased risk of recurrent MI and revascularization events after AMI [17]. 
TABLE 3: Subgroup analyses.

\begin{tabular}{|c|c|c|c|c|c|}
\hline \multicolumn{6}{|c|}{ Clinical presentation } \\
\hline & $\begin{array}{c}\text { Stable CAD and CABG } \\
\text { OR }(\mathrm{CI})\end{array}$ & $\begin{array}{l}\text { STEMI } \\
\text { OR (CI) }\end{array}$ & $P$ value & $\begin{array}{l}\text { NSTEMI } \\
\text { OR (CI) }\end{array}$ & $P$ value \\
\hline All-cause mortality & $0.87(0.71-1.07)$ & $0.89(0.59-1.34)$ & 0.92 & $2.47(0.47-13.08)$ & 0.22 \\
\hline Stroke & $0.97(0.60-1.55)$ & $1.06(0.69-1.62)$ & 0.78 & $2.90(0.12-72.20)$ & 0.51 \\
\hline Recurrent MI & $0.94(0.81-1.09)$ & $1.20(0.89-1.62)$ & 0.15 & $4.309(0.84-19.84)$ & 0.06 \\
\hline Revascularization & $0.93(0.82-1.09)$ & - & - & $1.060(0.65-3.95)$ & 0.78 \\
\hline MACCE & $0.81(0.65-1.01)$ & $1.02(0.91-1.13)$ & 0.07 & $1.29(0.94-1.77)$ & 0.02 \\
\hline \multicolumn{6}{|c|}{ Timing after acute event } \\
\hline & \multicolumn{2}{|c|}{$\leq 7$ days } & \multicolumn{3}{|c|}{$>7$ days } \\
\hline All-cause mortality & \multicolumn{2}{|c|}{$1.08(0.82-1.41)$} & \multicolumn{2}{|r|}{$0.94(0.87-1.02)$} & 0.33 \\
\hline Cardiac mortality & \multicolumn{2}{|c|}{$1.12(0.70-1.79)$} & \multicolumn{2}{|r|}{$0.92(0.83-1.02)$} & 0.42 \\
\hline Stroke & \multicolumn{2}{|c|}{$1.03(0.70-1.50)$} & \multicolumn{2}{|r|}{$0.90(0.70-1.16)$} & 0.56 \\
\hline Recurrent MI & \multicolumn{2}{|c|}{$1.34(0.97-1.86)$} & \multicolumn{2}{|r|}{$0.92(0.86-0.99)^{*}$} & 0.03 \\
\hline Revascularization & \multicolumn{2}{|c|}{$1.54(0.70-3.36)$} & \multicolumn{2}{|r|}{$0.83(0.71-0.98)^{*}$} & 0.03 \\
\hline MACCE & \multicolumn{2}{|c|}{$1.08(0.93-1.26)$} & \multicolumn{2}{|r|}{$0.91(0.82-1.00)$} & 0.06 \\
\hline \multicolumn{6}{|l|}{ Clinical presentation } \\
\hline & \multicolumn{2}{|c|}{ ACS } & \multicolumn{3}{|c|}{ Non-ACS } \\
\hline All-cause mortality & \multicolumn{2}{|c|}{$0.99(0.88-1.11)$} & \multicolumn{2}{|r|}{$0.87(0.71-1.07)$} & 0.28 \\
\hline Cardiac mortality & \multicolumn{2}{|c|}{$0.92(0.81-1.04)$} & \multicolumn{2}{|r|}{$0.94(0.80-1.10)$} & 0.84 \\
\hline Stroke & \multicolumn{2}{|c|}{$0.93(0.74-1.16)$} & \multicolumn{2}{|r|}{$0.97(0.60-1.55)$} & 0.88 \\
\hline Recurrent MI & \multicolumn{2}{|c|}{$1.08(0.91-1.27)$} & \multicolumn{2}{|r|}{$0.88(0.80-0.98)^{*}$} & 0.04 \\
\hline Revascularization & \multicolumn{2}{|c|}{$0.83(0.65-1.07)$} & \multicolumn{2}{|r|}{$0.93(0.82-1.05)$} & 0.42 \\
\hline MACCE & \multicolumn{2}{|c|}{$0.99(0.91-1.08)$} & \multicolumn{2}{|r|}{$0.83(0.68-1.01)$} & 0.11 \\
\hline
\end{tabular}

Although no definite explanation exists yet as per our knowledge, we believe it is possible that the timing and duration of drug administration may play a crucial role in determining response to medication.

4.3. Pexelizumab and Cardiovascular Outcomes. Our finding was consistent with a previous meta-analysis that included seven pexelizumab studies. They also did not find any significant improvements in major adverse cardiovascular outcomes and its components with the use of pexelizumab compared with placebo. However, they did find a $26 \%$ decreased risk of death in the CABG subpopulation (OR 0.74 [0.58-0.94]; $P=0.01$ ). It is hypothesized that the benefit seen in primarily CABG population may be related to the presence of intact microvascular system, salvageable myocardium, ability of the medication to penetrate the tissues, differences in the inflammatory pathway involved, and degree of complement system activation in this population as compared to the ACS population. Upstream delivery of pexelizumab in the STEMI population may not be effective as irreversible damage to the vascular system and myocardium prevents the penetration of medication to the site of inflammation yielding the drug less effective [48].

\section{Study Limitations}

There were some limitations to this study. First, although we included all patients with coronary artery disease, they range in severity of clinical presentation from stable coronary heart disease to acute coronary syndrome requiring revascularization procedure (PCI or CABG). This heterogeneity could explain some of our findings and we aimed to address them by conducting extensive subgroup analyses. Second, there is variability in the length of follow-up among trials from 30 days to 3.7 years. Third, the three anakinra trials that were included in our analysis are small studies with less than 200 patients. Although we see a potential harm with the use of anakinra (increased risk of recurrent MI), the potential bias with the small study sample must not be overlooked. Further larger anakinra studies will be required to explore this interesting association. Fourth, the trials of two medications in our study (pexelizumab and varespladib) did not exactly have the same intervention protocol. Two out of the six pexelizumab trials (COMMA and COMPLY) were given an infusion of only 20 hours as opposed to the 24-hour duration studies in the rest of the studies. Additionally, although both varespladib trials (FRANCIS and VISTA-16 trials) utilized the same dose of varespladib, the duration of treatment and the statin dose are different ( 24 vs. 16 weeks and $80 \mathrm{mg}$ vs. at least $20 \mathrm{mg}$, respectively).

Despite these limitations, our analysis has several strengths. To our knowledge, this is the first study to assess the cumulative efficacy of eight different anti-inflammatory medications and compare their individual efficacy on cardiovascular outcomes of patients with coronary artery disease. Second, our study was also the first to analyze the efficacy 
of anti-inflammatory medication based on timing of drug administration and patient's clinical presentation. Third, our outcomes of interest (e.g., death, CV death, and MI) are largely objective findings and most of the trials included in our study have independent event adjudicators, thus minimizing the risk of measurement bias.

\section{Conclusion}

When applied to a largely unselected patient population with coronary artery disease, anti-inflammatory medications failed to reduce adverse cardiovascular outcomes. However, selected agents show promise among subgroups of patients without ACS or after the first week following an acute ischemic event. Future studies examining the proper timing and targetable inflammatory pathways are warranted.

\section{Conflicts of Interest}

The authors declare that they have no conflicts of interest.

\section{Authors' Contributions}

Ivan Wudexi and Elica Shokri contributed equally to the work.

\section{Acknowledgments}

Ahmed Abdel-Latif is supported by the NIH R01 grant \# HL124266.

\section{Supplementary Materials}

Supplemental Table 1: bias risk assessment of the studies. Supplemental Figure 1: forest plot for network metaanalysis comparing the relative efficacy of each antiinflammatory medication on all-cause death. Use of pexelizumab is associated with lower risk of all-cause mortality in comparison with veraspladib (OR 0.62, CI 0.37-0.99). Supplemental Figure 2: forest plot for network meta-analysis comparing the relative efficacies of each anti-inflammatory medication on cardiovascular death. Supplemental Figure 3: forest plot for network meta-analysis of myocardial infarction. Uses of canakinumab, colchicine, darapladib, and pexelizumab were associated with lower risk of recurrent myocardial infarction in comparison with anakinra (OR 0.20, CI 0.04-0.79; OR 0.21, CI 0.04-0.83; OR 0.22, CI 0.04-0.81; and OR 0.22, CI 0.05-0.82, respectively). Supplemental Figure 4: forest plot for network meta-analysis of revascularization. Use of colchicine significantly reduced the risk of revascularization in comparison to both anakinra and darapladib (OR 0.31, CI 0.11-0.84 and OR 0.52, CI 0.290.93 , respectively). Supplemental Figure 5: forest plot for network meta-analysis of stroke. Use of colchicine was associated with significant reduced risk of stroke events after myocardial infarction in comparison to several antiinflammatory medications including: darapladib (OR 0.23 , CI 0.07-0.57), pexelizumab (OR 0.23, CI 0.07-0.64), losmapimod (OR 0.25, CI 0.07-0.85), canakinumab (OR 0.30, CI 0.09-0.81), and veraspladib (OR 0.26, CI 0.07-0.97). Supple- mental Figure 6: forest plot for network meta-analysis comparing the relative efficacy of each anti-inflammatory medication on major adverse cardiac and cerebrovascular events (MAACE). Colchicine use was associated with significantly lower risk of MACCE when compared to darapladib (OR 0.69, CI 0.44-0.98), losmapimod (OR 0.60, CI 0.370.93 ), anakinra (OR $0.28 \mathrm{CI} 0.10-0.70$ ), and varespladib (OR 0.53, CI 0.32-0.83). Both canakinumab and pexelizumab were associated with reduced risk of MACCE (OR 0.37, CI 0.13-0.95 and OR 0.39, CI 0.14-0.96, respectively). Supplemental Figure 7: network plot of treatments included in this network meta-analysis. Circles represent the intervention as a node in the network, lines represent direct comparisons using randomized clinical trials (RCTs), and the thickness of lines corresponds to the number of (RCTs) included in each comparison. Supplemental Figure 8: funnel plots of odds ratios and standard errors to assess the publication bias of all-cause mortality, cardiac mortality, revascularization, stroke, recurrent myocardial infarction, and major adverse cardiac and cerebrovascular events (MACCE). Supplemental Figure 9: consistency plot for all-cause mortality, cardiac mortality, revascularization, stroke, recurrent myocardial infarction, and major adverse cardiac and cerebrovascular events (MACCE). (Supplementary Materials)

\section{References}

[1] A. S. Go, D. Mozaffarian, V. L. Roger et al., "Heart disease and stroke statistics-2014 update: a report from the American Heart Association," Circulation, vol. 129, no. 3, pp. e28-e292, 2014.

[2] C. W. Hamm, J. P. Bassand, S. Agewall et al., "ESC guidelines for the management of acute coronary syndromes in patients presenting without persistent ST-segment elevation: the task force for the management of acute coronary syndromes (ACS) in patients presenting without persistent ST-segment elevation of the European Society of Cardiology (ESC)," European Heart Journal, vol. 32, no. 23, pp. 2999-3054, 2011.

[3] L. K. Newby, M. S. Marber, C. Melloni et al., "Losmapimod, a novel p38 mitogen-activated protein kinase inhibitor, in nonST- segment elevation myocardial infarction: a randomised phase 2 trial," Lancet, vol. 384, no. 9949, pp. 1187-1195, 2014.

[4] S. Epelman, P. P. Liu, and D. L. Mann, "Role of innate and adaptive immune mechanisms in cardiac injury and repair," Nature Reviews. Immunology, vol. 15, no. 2, pp. 117-129, 2015.

[5] K. E. Berg, I. Ljungcrantz, L. Andersson et al., "Elevated CD14 ++CD16- monocytes predict cardiovascular events," Circulation. Cardiovascular Genetics, vol. 5, no. 1, pp. 122-131, 2012.

[6] Y. Maekawa, T. Anzai, T. Yoshikawa et al., "Prognostic significance of peripheral monocytosis after reperfused acute myocardial infarction:a possible role for left ventricular remodeling," Journal of the American College of Cardiology, vol. 39, no. 2, pp. 241-246, 2002.

[7] P. Panizzi, F. K. Swirski, J. L. Figueiredo et al., "Impaired Infarct Healing in Atherosclerotic Mice With Ly-6Chi Monocytosis," Journal of the American College of Cardiology, vol. 55, no. 15, pp. 1629-1638, 2010.

[8] N. Ruparelia, J. Godec, R. Lee et al., “Acute myocardial infarction activates distinct inflammation and proliferation pathways in circulating monocytes, prior to recruitment, 
and identified through conserved transcriptional responses in mice and humans," European Heart Journal, vol. 36, no. 29, pp. 1923-1934, 2015.

[9] A. al-Darraji, D. Haydar, L. Chelvarajan et al., "Azithromycin therapy reduces cardiac inflammation and mitigates adverse cardiac remodeling after myocardial infarction: potential therapeutic targets in ischemic heart disease," PLoS One, vol. 13, no. 7, article e0200474, 2018.

[10] T. Ben-Mordechai, D. Palevski, Y. Glucksam-Galnoy, I. ElronGross, R. Margalit, and J. Leor, "Targeting macrophage subsets for infarct repair," Journal of Cardiovascular Pharmacology and Therapeutics, vol. 20, no. 1, pp. 36-51, 2014.

[11] G. de Couto, W. Liu, E. Tseliou et al., "Macrophages mediate cardioprotective cellular postconditioning in acute myocardial infarction," The Journal of Clinical Investigation, vol. 125, no. 8, pp. 3147-3162, 2015.

[12] N. G. Frangogiannis, "The inflammatory response in myocardial injury, repair, and remodelling," Nature Reviews. Cardiology, vol. 11, no. 5, pp. 255-265, 2014.

[13] B. H. Bulkley and W. C. Roberts, "Steroid therapy during acute myocardial infarction: A cause of delayed healing and of ventricular aneurysm," The American Journal of Medicine, vol. 56, no. 2, pp. 244-250, 1974.

[14] A. M. Schjerning Olsen, E. L. Fosbøl, J. Lindhardsen et al., "Duration of treatment with nonsteroidal anti-inflammatory drugs and impact on risk of death and recurrent myocardial infarction in patients with prior myocardial Infarction," Circulation, vol. 123, no. 20, pp. 2226-2235, 2011.

[15] M. Horckmans, L. Ring, J. Duchene et al., "Neutrophils orchestrate post-myocardial infarction healing by polarizing macrophages towards a reparative phenotype," European Heart Journal, vol. 38, no. 3, pp. 187-197, 2016.

[16] C. B. Granger, K. W. Mahaffey, W. D. Weaver et al., "Pexelizumab, an anti-C5 complement antibody, as adjunctive therapy to primary percutaneous coronary intervention in acute myocardial infarction," Circulation, vol. 108, no. 10, pp. 11841190, 2003.

[17] P. M. Ridker, B. M. Everett, T. Thuren et al., "Antiinflammatory therapy with canakinumab for atherosclerotic disease," The New England Journal of Medicine, vol. 377, no. 12, pp. 1119-1131, 2017.

[18] P. M. Ridker, J. G. MacFadyen, B. M. Everett et al., "Relationship of C-reactive protein reduction to cardiovascular event reduction following treatment with canakinumab: a secondary analysis from the CANTOS randomised controlled trial," Lancet, vol. 391, no. 10118, pp. 319-328, 2018.

[19] J. A. Rymer and L. K. Newby, "Failure to launch: targeting inflammation in acute coronary syndromes," JACC Basic Transl Sci, vol. 2, no. 4, pp. 484-497, 2017.

[20] L. Shamseer, D. Moher, M. Clarke et al., "Preferred reporting items for systematic review and meta-analysis protocols (PRISMA-P) 2015: elaboration and explanation," BMJ, vol. 349, no. jan02 1, article g7647, 2015.

[21] J. P. Higgins, D. G. Altman, P. C. Gotzsche et al., "The Cochrane Collaboration's tool for assessing risk of bias in randomised trials," BMJ, vol. 343, no. oct18 2, article d5928, 2011.

[22] R. Der Simonian and N. Laird, "Meta-analysis in clinical trials," Controlled Clinical Trials, vol. 7, no. 3, pp. 177-188, 1986.

[23] J. P. Higgins, S. G. Thompson, J. J. Deeks, and D. G. Altman, "Measuring inconsistency in meta-analyses," BMJ, vol. 327, no. 7414, pp. 557-560, 2003.
[24] T. B. Huedo-Medina, J. Sánchez-Meca, F. Marín-Martínez, and J. Botella, "Assessing heterogeneity in meta-analysis: Q statistic or $\mathrm{I}^{2}$ index?," Psychological Methods, vol. 11, no. 2, pp. 193-206, 2006.

[25] D. M. Caldwell, A. E. Ades, and J. P. Higgins, "Simultaneous comparison of multiple treatments: combining direct and indirect evidence," BMJ, vol. 331, no. 7521, pp. 897-900, 2005.

[26] S. Brown, B. Hutton, T. Clifford et al., "A Microsoft-Excelbased tool for running and critically appraising network meta-analyses-an overview and application of NetMetaXL," Systematic Reviews, vol. 3, no. 1, p. 110, 2014.

[27] S. Dias, N. J. Welton, A. J. Sutton, D. M. Caldwell, G. Lu, and A. E. Ades, "Evidence synthesis for decision making 4: inconsistency in networks of evidence based on randomized controlled trials," Medical Decision Making, vol. 33, no. 5, pp. 641-656, 2013.

[28] APEX AMI Investigators, P. W. Armstrong, C. B. Granger et al., "Pexelizumab for acute ST-elevation myocardial infarction in patients undergoing primary percutaneous coronary Intervention," JAMA, vol. 297, no. 1, pp. 43-51, 2007.

[29] K. W. Mahaffey, C. B. Granger, J. C. Nicolau et al., "Effect of pexelizumab, an anti-C5 complement antibody, as adjunctive therapy to fibrinolysis in acute myocardial infarction," Circulation, vol. 108, no. 10, pp. 1176-1183, 2003.

[30] S. K. Shernan, J. C. Fitch, N. A. Nussmeier et al., "Impact of pexelizumab, an anti-C5 complement antibody, on total mortality and adverse cardiovascular outcomes in cardiac surgical patients undergoing cardiopulmonary bypass," The Annals of Thoracic Surgery, vol. 77, no. 3, pp. 942-949, 2004.

[31] P. K. Smith, S. K. Shernan, J. C. Chen et al., "Effects of C5 complement inhibitor pexelizumab on outcome in high-risk coronary artery bypass grafting: combined results from the PRIMO-CABG I and II trials," The Journal of Thoracic and Cardiovascular Surgery, vol. 142, no. 1, pp. 89-98, 2011.

[32] E. D. Verrier, S. K. Shernan, K. M. Taylor et al., "Terminal complement blockade with pexelizumab during coronary artery bypass graft surgery requiring cardiopulmonary Bypass," JAMA, vol. 291, no. 19, pp. 2319-2327, 2004.

[33] A. Abbate, M. C. Kontos, N. A. Abouzaki et al., "Comparative Safety of Interleukin-1 Blockade With Anakinra in Patients With ST-Segment Elevation Acute Myocardial Infarction (from the VCU-ART and VCU- ART2 Pilot Studies)," The American Journal of Cardiology, vol. 115, no. 3, pp. 288-292, 2015.

[34] A. Abbate, C. R. Trankle, L. F. Buckley et al., "Interleukin-1 blockade inhibits the acute inflammatory response in patients with ST-segment-elevation myocardial infarction," Journal of the American Heart Association, vol. 9, no. 5, article e014941, 2020.

[35] A. C. Morton, A. M. Rothman, J. P. Greenwood et al., "The effect of interleukin-1 receptor antagonist therapy on markers of inflammation in non-ST elevation acute coronary syndromes: the MRC-ILA Heart Study," European Heart Journal, vol. 36, no. 6, pp. 377-384, 2015.

[36] S. M. Nidorf, J. W. Eikelboom, C. A. Budgeon, and P. L. Thompson, "Low-dose colchicine for secondary prevention of cardiovascular disease," Journal of the American College of Cardiology, vol. 61, no. 4, pp. 404-410, 2013.

[37] J. C. Tardif, S. Kouz, D. D. Waters et al., "Efficacy and safety of low-dose colchicine after myocardial infarction," The New England Journal of Medicine, vol. 381, no. 26, pp. 2497-2505, 2019. 
[38] M. L. O'Donoghue, E. Braunwald, H. D. White et al., "Effect of darapladib on major coronary events after an acute coronary syndrome: the SOLID-TIMI 52 randomized clinical trial," JAMA, vol. 312, no. 10, pp. 1006-1015, 2014.

[39] STABILITY Investigators, H. D. White, C. Held et al., "Darapladib for preventing ischemic events in stable coronary heart disease," The New England Journal of Medicine, vol. 370, no. 18, pp. 1702-1711, 2014.

[40] S. J. Nicholls, J. J. Kastelein, G. G. Schwartz et al., "Varespladib and cardiovascular events in patients with an acute coronary syndrome: the VISTA-16 randomized clinical trial," JAMA, vol. 311, no. 3, pp. 252-262, 2014.

[41] R. S. Rosenson, C. Hislop, M. Elliott, Y. Stasiv, M. Goulder, and D. Waters, "Effects of varespladib methyl on biomarkers and major cardiovascular events in acute coronary syndrome patients," Journal of the American College of Cardiology, vol. 56, no. 14, pp. 1079-1088, 2010.

[42] B. E. Stähli, J. C. Tardif, M. Carrier et al., "Effects of P-selectin antagonist inclacumab in patients undergoing coronary artery bypass graft surgery: SELECT-CABG trial," Journal of the American College of Cardiology, vol. 67, no. 3, pp. 344-346, 2016.

[43] M. L. O’Donoghue, R. Glaser, M. A. Cavender et al., "Effect of losmapimod on cardiovascular outcomes in patients hospitalized with acute myocardial infarction: a randomized clinical trial,” JAMA, vol. 315, no. 15, pp. 1591-1599, 2016.

[44] A. H. Katsanos, L. Palaiodimou, C. Price et al., "Colchicine for stroke prevention in patients with coronary artery disease: a systematic review and meta-analysis," European Journal of Neurology, vol. 27, no. 6, pp. 1035-1038, 2020.

[45] K. Vaidya, C. Arnott, G. J. Martínez et al., "Colchicine therapy and plaque stabilization in patients with acute coronary syndrome: a CT coronary angiography study," JACC: Cardiovascular Imaging, vol. 11, no. 2 Part 2, pp. 305-316, 2018.

[46] G. J. Martínez, S. Robertson, J. Barraclough et al., "Colchicine acutely suppresses local cardiac production of inflammatory cytokines in patients with an acute coronary syndrome," Journal of the American Heart Association, vol. 4, no. 8, article e002128, 2015.

[47] Z. H. Zheng, X. Zeng, X. Y. Nie et al., "Interleukin-1 blockade treatment decreasing cardiovascular risk," Clinical Cardiology, vol. 42, no. 10, pp. 942-951, 2019.

[48] L. Testa, W. J. van Gaal, R. Bhindi et al., "Pexelizumab in ischemic heart disease: a systematic review and meta-analysis on 15,196 patients," The Journal of Thoracic and Cardiovascular Surgery, vol. 136, no. 4, pp. 884-893, 2008. 